\title{
Radiation protection in dental X-ray surgeries - still rooms for improvement
}

\author{
G. Hart ${ }^{* 1}$ and M. Dugdale ${ }^{2}$ \\ CORE VERIFIABLE CPD PAPER
}

Aim To illustrate the authors' experience in the provision of radiation protection adviser (RPA)/medical physics expert (MPE) services and critical examination/radiation quality assurance (QA) testing, to demonstrate any continuing variability of the compliance of $\mathrm{X}$-ray sets with existing guidance and of compliance of dental practices with existing legislation. Method Data was collected from a series of critical examination and routine three-yearly radiation QA tests on 915 intraoral X-ray sets and 124 panoramic sets. Data are the result of direct measurements on the sets, made using a traceably calibrated Unfors Xi meter. The testing covered the measurement of peak kilovoltage ( $\mathrm{V} V \mathrm{p})$; filtration; timer accuracy and consistency; $X$-ray beam size; and radiation output, measured as the entrance surface dose in milliGray (mGy) for intraoral sets and dose-area product (DAP), measured in $\mathrm{mGy} \cdot \mathrm{cm}^{2}$ for panoramic sets. Physical checks, including mechanical stability, were also included as part of the testing process. Results The Health and Safety Executive has expressed concern about the poor standards of compliance with the regulations during inspections at dental practices. Thirty-five percent of intra-oral sets exceeded the UK adult diagnostic reference level on at least one setting, as did 61\% of those with child dose settings. There is a clear advantage of digital radiography and rectangular collimation in dose terms, with the mean dose from digital sets 59\% that of film-based sets and a rectangular collimator 76\% that of circular collimators. The data shows the unrealised potential for dose saving in many digital sets and also marked differences in dose between sets. Conclusion Provision of radiation protection advice to over 150 general dental practitioners raised a number of issues on the design of surgeries with $\mathrm{X}$-ray equipment and critical examination testing. There is also considerable variation in advice given on the need (or lack of need) for room shielding. Where no radiation protection adviser (RPA) or medical physics expert (MPE) appointment has been made, there is often a very low level of compliance with legislative requirements. The active involvement of an RPA/MPE and continuing education on radiation protection issues has the potential to reduce radiation doses significantly further in many dental practices.

\section{INTRODUCTION}

The Health and Safety Executive's (HSE) Radiation Protection News of June $2010^{1}$ stated that their radiation team were 'concerned about the poor standards of compliance with the IRR99 they have found during inspections at Dental Practices and Chiropractors. Many fail to properly comply with the IRR99' (Ionising Radiations Regulations 1999)' 'and some have not even paid scant attention to its most basic requirements. As a result they have put

${ }^{1}$ YourRPA - Independent Radiation and Laser Protection Adviser, 96 Sefton Road, Morecambe, Lancashire, LA3 IUD; ${ }^{2}$ Radiation QA Services Limited, Thorniehurst, The Woodlands, Roundwood Road, Baildon, BD17 6SP

${ }^{*}$ Correspondence to: Graham Hart

Email:YourRPA@yahoo.co.uk

Online article number E16

Refereed Paper - accepted 7 December 2012

DOI: 10.1038/sj.bdj.2013.274

${ }^{\circ}$ British Dental Journal 2013; 214: E16 themselves, their staff and members of the public to unnecessary risk.' ${ }^{1}$ The newsletter specifically mentioned a number of issues, including the production of a 'suitable and sufficient' radiation risk assessment, training; appointment of a radiation protection adviser (RPA), production of local rules and the maintenance of $\mathrm{X}$-ray equipment.

Given that practices which have not appointed an RPA have shown a low level of compliance with IRR99, there is reason to believe that if they have not appointed a medical physics expert (MPE) they would also be likely to show a low level of compliance with the Ionising Radiation (medical exposure) Regulations 2000 (as amended 2006 and 2011) (IRMER) ${ }^{3}$ despite the publication of detailed guidance notes on compliance with both IRR99 and IRMER back in 2001. ${ }^{4}$

Latest data from the Health Protection Agency (HPA) ${ }^{5}$ shows that dental X-ray examinations remain the most common radiological investigation, with almost 12 million investigations taking place in 2008, representing a dental X-ray in almost one in five of the population. Although dental X-ray examinations are the most frequent, the same report demonstrates that they do not appear in the 20 types of examination that contribute most to the total collective dose. This is because the effective dose from most dental X-ray examinations is low, as demonstrated by the last HPA report on dental radiation doses to patients published in $2007 .^{6}$

However, the introduction of more complex dental radiographic techniques, such as cone beam computed tomography (CBCT), does have the potential to radically increase patient exposure, as outlined by the report from the HPA Working Party on 
dental cone beam $\mathrm{CT}^{7}$ and their subsequent guidance document. ${ }^{8}$

The aim of this paper is to illustrate the authors' experience in the provision of RPA/ MPE services and critical examination/ radiation quality assurance $(\mathrm{QA})$ testing, to demonstrate any continuing variability of the compliance of X-ray sets with existing guidance and of compliance of dental practices with existing legislation.

\section{MATERIALS AND METHODS}

Data has been collected from a series of critical examination and routine three-yearly radiation QA tests on 915 intra-oral X-ray sets and 124 panoramic sets. Unlike the HPA 'postal pack' system, this data is the result of direct measurements on the sets, made using a traceably calibrated Unfors Xi meter. The testing covered the measurement of peak kilovoltage $(\mathrm{kVp})$; filtration; timer accuracy and consistency; X-ray beam size; and radiation output, measured as the entrance surface dose in milliGray (mGy) for intra-oral sets and dose-area product (DAP), measured in $\mathrm{mGy} . \mathrm{cm}^{2}$ for panoramic sets. Physical checks, including mechanical stability, were also included as part of the testing process.

Maximum radiation output was assessed against the UK's National Reference Dose (NRD), a form of Diagnostic Reference Level, defined in IRMER as dose levels in radiodiagnostic practices for typical examinations for groups of standardsized patients or standard phantoms for broadly defined types of equipment. Recommendations for dental NRDs are provided by the HPA. ${ }^{6}$ The dental guidance notes suggest that "they would not normally be expected to be exceeded without good reason'.

Data has also been collected from the provision of RPA/MPE services to 136 general dental practices, having a total of 317 intra-oral X-ray sets and 41 panoramic sets. Information was obtained by questionnaire regarding:

- X-ray equipment, to capture the data required by IRMER

- Room layout and wall construction, to assess what level of shielding was in place

- Position of the X-ray control box and isolator switch, to assess whether any radiation hazard might be created in the event of the set failing to terminate its exposure

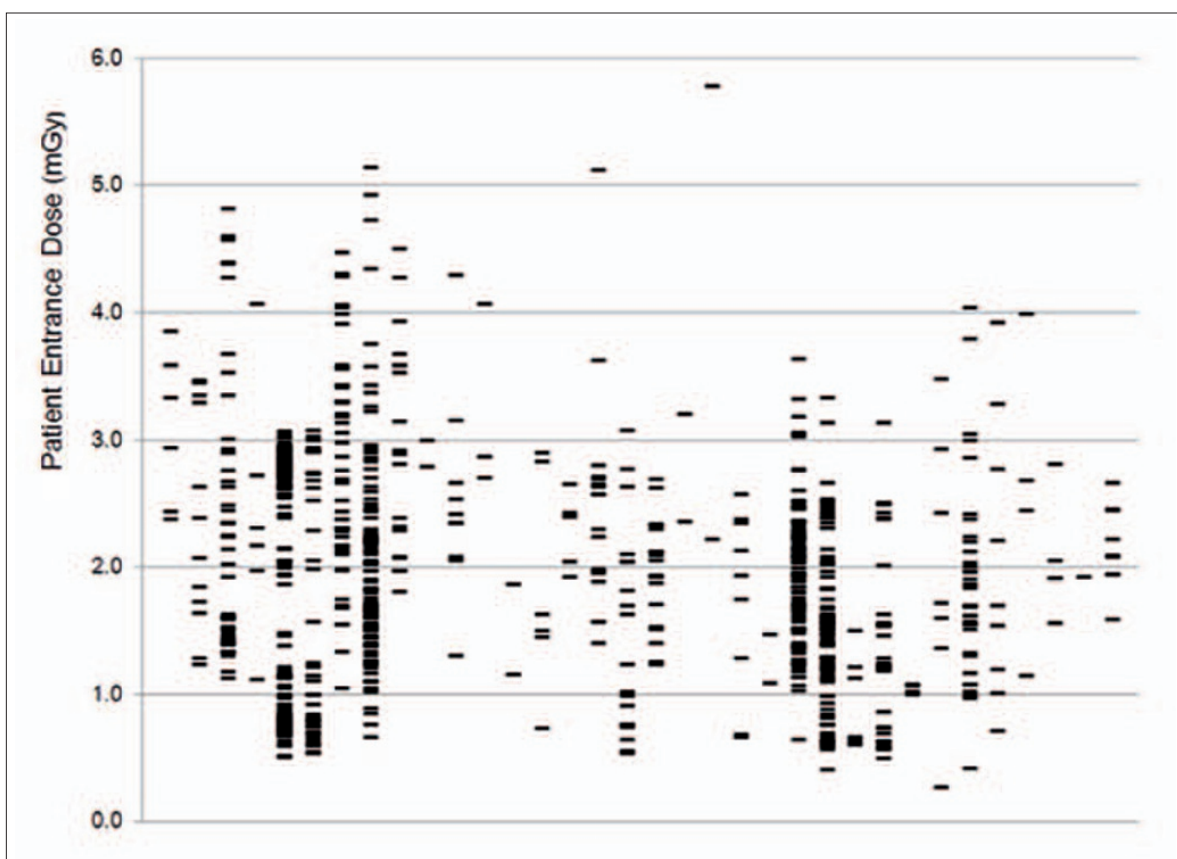

Fig. 1 Maximum patient entrance surface doses for different manufacturers and models of intra-oral sets. Each column indicates a particular model of X-ray set and each short horizontal bar a single measurement

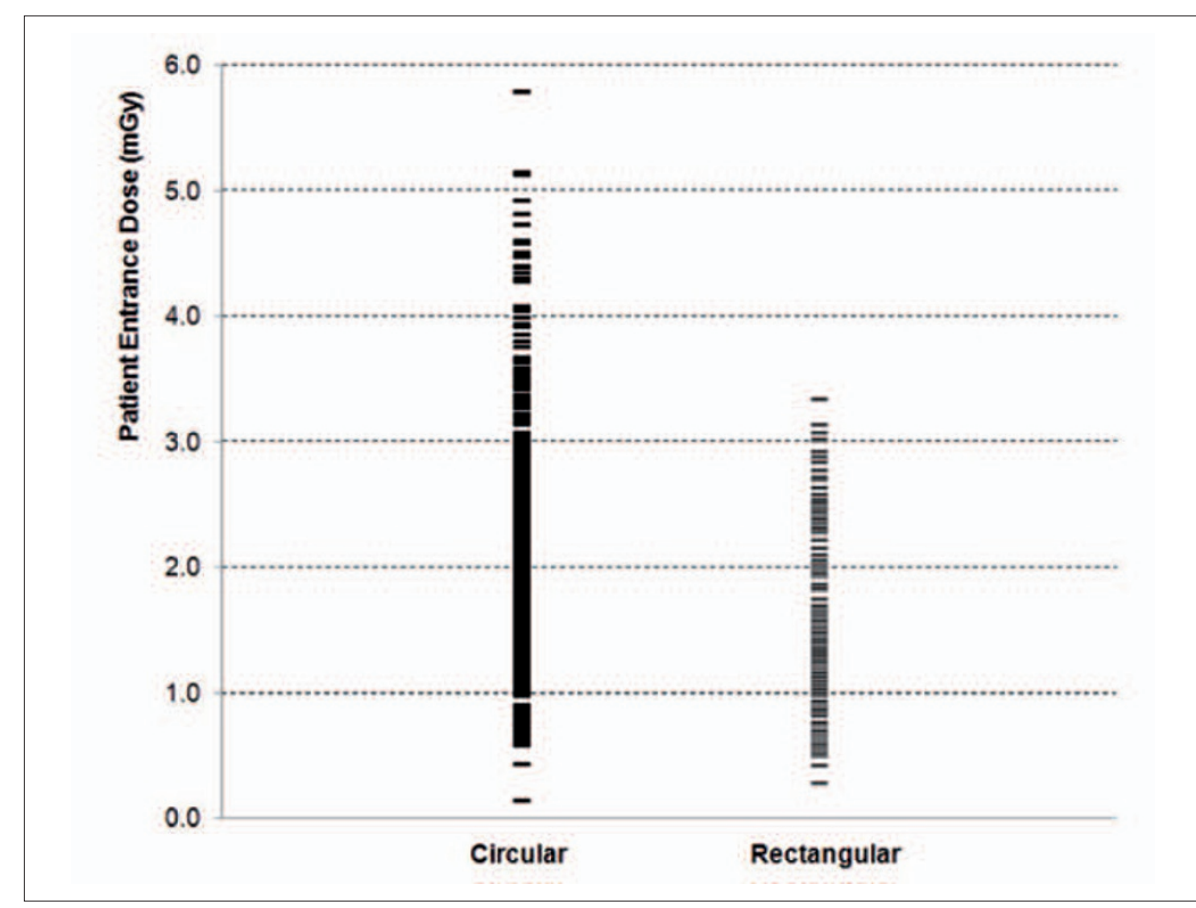

Fig. 2 Maximum patient entrance surface doses for intra-oral sets with circular and rectangular collimators

- Operator's position when effecting $\mathrm{X}$-ray exposures, to ensure they remain outside the radiation controlled area and ideally at least two metres from the patient during exposures, as well as away from the line of the main $\mathrm{X}$-ray beam

- Whether digital or film-based imaging was used

- Whether staff were monitored for radiation dose, and if so, to provide recent dose data

- The frequency of X-ray use in terms of average number of exposures per week, to assess staff radiation exposure.

This was supplemented by visits to approximately $10 \%$ of clients, either at their request or where $\mathrm{CBCT}$ equipment was either planned or installed. 


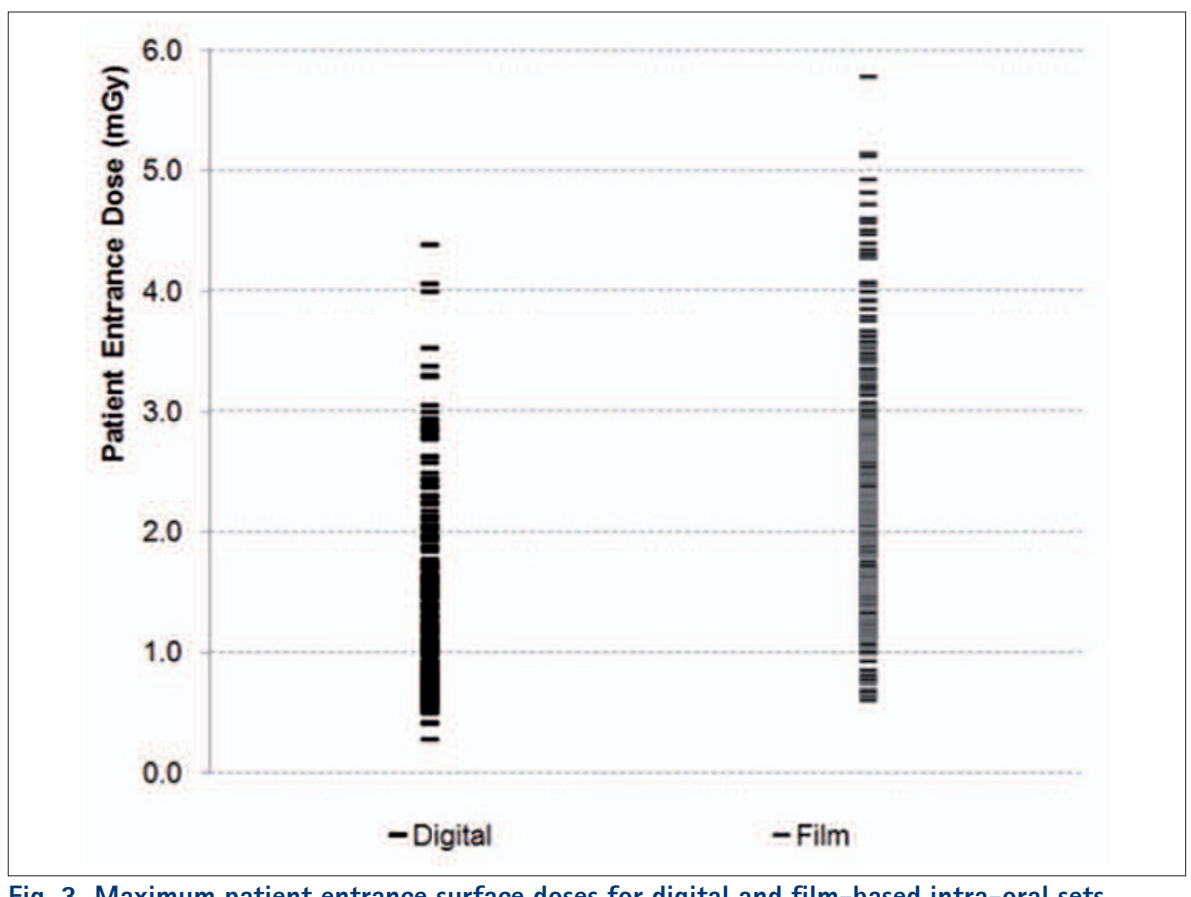

Fig. 3 Maximum patient entrance surface doses for digital and film-based intra-oral sets

All critical examination/QA measurements and RPA/MPE documentation and visits occurred during the period 2008-2012. Approximately 90\% of the critical examination/QA data originate from dental practices in northern England, covering an area from Derbyshire to the Scottish border, with the remainder coming from London and South-East England. The RPA/MPE data is from dental practices over the whole of England (with two practices in Scotland), although again with a prominence from the north.

While there is significant overlap between the datasets for the dental practices covered by the critical examination/ QA checks and the RPA/MPE service, some practices are unique to each dataset.

\section{RESULTS}

\section{Radiation critical examination and QA tests}

Only 2 of the 915 intra-oral X-ray sets tested still operated at $50 \mathrm{kVp}$. All others operated at a minimum of $60 \mathrm{kVp}$.

All sets tested (both intra-oral and panoramic) met the minimum requirement for filtration of $1.5 \mathrm{~mm}$ aluminium for sets operating at up to and including $70 \mathrm{kVp}$, or $2.0 \mathrm{~mm}$ aluminium for sets operating at tube voltages above $70 \mathrm{kVp}$.

All intra-oral sets met the recommendation for maximum beam size of $60 \mathrm{~mm}$ diameter for those with circular collimators.
The range of circular collimator diameters within the survey was $54-60 \mathrm{~mm}$, with a mean of $59 \mathrm{~mm}$. Twenty-five percent of the intra-oral sets tested were equipped with rectangular collimators, all of which were within (but generally at) the maximum recommended size of $35 \mathrm{~mm} \times 45 \mathrm{~mm}$. Only three panoramic sets exceeded the recommended maximum beam size of $5 \mathrm{~mm} \times 150 \mathrm{~mm}$.

For panoramic sets, 296 DAP measurements were made at different $\mathrm{kV} / \mathrm{mA}$ settings on a total of 124 panoramic sets. DAP values ranged from 7-179 mGy.cm², with a mean DAP value of $58.8 \mathrm{mGy} . \mathrm{cm}^{2}$ and a median of $54.5 \mathrm{mGy} . \mathrm{cm}^{2}$. Eighteen percent of the panoramic sets had at least one measurement above the current UK NRD of 82 mGy.cm².

Measured maximum entrance doses for intra-oral sets ranged from $0.13 \mathrm{mGy}$ $6.53 \mathrm{mGy}$, with a mean dose of $1.96 \mathrm{mGy}$ and a median dose of 1.93 mGy. Thirtyfive percent of sets exceeded the adult NRD of 2.3 mGy given in HPA Report $029^{6}$ on at least one setting. Forty percent of those sets with a child dose setting exceeded the NRD of 1.5 mGy on at least one setting.

Maximum intra-oral radiation doses varied widely from manufacturer to manufacturer, and for different models of any given manufacturer, as demonstrated in Figure 1.

When the same data is plotted in terms of the collimator shape (Fig. 2), it reveals a generally lower radiation dose for sets with rectangular collimators. Measured maximum doses for sets with circular collimators ranged from 0.15$6.54 \mathrm{mGy}$ (mean $\pm \mathrm{SD}=2.05 \pm 0.93 \mathrm{mGy}$, where $\mathrm{SD}$ is the standard deviation), whereas for sets with rectangular collimators it ranged from 0.28-3.34 mGy $($ mean \pm SD $=1.55 \pm 0.69 \mathrm{mGy})$. The mean dose for sets with rectangular collimators was thus 76\% that of sets with circular collimators, the difference being statistically significant ( $\mathrm{p}<0.001)$.

When comparing the maximum dose from digital and film-based X-ray sets, this ranged from 0.28-4.79 $\mathrm{mGy}$ (mean $\pm \mathrm{SD}=1.31 \pm 0.73 \mathrm{mGy}$ ) for digital sets and from 0.7-6.54 mGy (mean $\pm \mathrm{SD}=2.22 \pm 0.82 \mathrm{mGy}$ ) for filmbased sets. The mean digital dose was therefore 59\% of that from film-based sets. This data is shown graphically in Figure 3, the difference again being statistically significant ( $p<0.001)$. The data also reveals that only $10.7 \%$ of digital sets exceed the adult NRD on at least one setting, compared to $85.7 \%$ of film-based ones. For sets with child dose settings, $24.2 \%$ of digital sets exceed the NRD on at least one setting, compared to $50.8 \%$ of film-based ones.

Timers on intra-oral sets were consistent but frequently inaccurate. Timer errors ranged from 0-500\%, with 37\% of sets with marked set times having errors greater than $10 \%$.

\section{RPA/MPE issues}

Of the 317 intra-oral X-ray sets in the 136 dental practices, 135 sets used filmbased imaging systems and 182 used digital imaging. One hundred and ninety-four sets used circular collimators and 123 used rectangular collimators. All collimators were within the maximum dimensions recommended in the dental guidance notes. ${ }^{3}$

One hundred and seven of the 317 intraoral X-ray sets (34\%) were advised that the radiation doses their patients received had a least one setting that exceeded the NRD. Of these 107 sets, 40 (37\%) were advised that dose reductions of at least a factor of two should be possible without reducing image quality to non-diagnostic levels. Four of the panoramic sets had at least one setting above the NRD.

The frequency of X-ray exposures ranged from 2-150 per week per set, with a mean value of 33 exposures per week 
per set. Both the median and modal values were 30 exposures per week per set. Ten X-ray sets had a usage of $\geq 100$ exposures per week per set.

Only two dental practices monitored their staff for radiation exposure, with both obtaining results below the UK's national mean value of $0.08 \mathrm{mSv} \cdot \mathrm{y}^{-1}$ for dental staff.

Twelve dental practices had installed 'lead lining' to one or more walls within the practice, with two of those dental practices also having installed lead lined doors before the appointment of the author as RPA.

Twenty-one of the 317 intra-oral X-ray sets required advice to amend the operator position during radiographic exposures, as they were either potentially standing in the line of the main X-ray beam for certain exposures or were in positions where the scattered radiation dose meant that they might not be receiving radiation doses that were as low as reasonably practicable. In nine cases the position of the isolator switch was sub-optimal, in that in the event of a set failing to terminate an exposure, the operator might have to enter the radiation field in order to isolate the set from the mains.

\section{DISCUSSION}

\section{Radiation critical examination and $\mathrm{QA}$ tests}

Both film-based and digital X-ray sets show a wide variation in maximum radiation doses. Four models of X-ray set in this survey always had some doses above the DRL. However, most of the variation cannot be attributed to the type or model of X-ray set used, since Figure 1 demonstrates that the variation exists for almost any given manufacturer or model of X-ray set within the survey. The wide range of maximum doses for both filmbased and digital sets demonstrates that there remains scope for significant dose reduction in many dental practices.

The data for digital sets in Figure 3, with a mean maximum radiation dose $60 \%$ that of film-based sets, clearly demonstrates the dose saving that can easily be achieved by using digital sets. However, it also illustrates the often unrealised potential for dose saving using these technologies. In some cases, dental practices have changed from film-based to digital receptors but have simply continued to use their existing film settings and where those were already sub-optimal a significant opportunity for dose reduction has been missed.

Some of the variation in recorded doses for digital sets may be due to the difference in exposure latitude of either direct digital plates (also known as direct digital radiography or DR) or phosphor plates (also known as computed radiography or $\mathrm{CR}$ ). Similarly some of the variation of filmbased sets may well have been due to differences in film speed used. This level of information was not recorded in the survey and thus cannot be analysed here.

The data regarding choice of collimation shows that the mean maximum radiation doses from sets with rectangular collimation was $76 \%$ that of those with circular collimation, a mean dose saving of $24 \%$. Although this is smaller than might be expected from a pure ratio of beam areas $\left(59 \mathrm{~mm}\right.$ diameter $=2734 \mathrm{~mm}^{2}$, $35 \mathrm{~mm} \times 45 \mathrm{~mm}=1575 \mathrm{~mm}^{2}$, ratio $=0.58$ ), it nevertheless demonstrates the value of using rectangular collimation, as recommended in the dental guidance notes. ${ }^{4}$ Given that $75 \%$ of the sets within the critical examination/QA survey and 60\% of sets within the RPA/MPE survey were still using circular collimation, the data again suggests a significant potential for further dose reduction. It is understood that there are some technical and practical difficulties with the introduction and use of rectangular collimators on some models of dental X-ray set. Nevertheless, given the potential dose saving, dental practices should continue to be encouraged to change to rectangular collimation whenever practicable.

\section{RPA/MPE issues}

Practices that had not previously employed an RPA or MPE demonstrated a low level of compliance with both IRR99 and IRMER. This occurred because basic regulatory compliance issues such as the production of radiation risk assessments, local rules, quality assurance procedures, and the raft of policies and procedures required by the IRMER and detailed in the Dental Guidance Notes had not been addressed.

Apart from this, two key issues have dominated: critical examination/routine QA testing for X-rays sets and design/ shielding issues for dental surgeries containing $\mathrm{X}$-ray equipment.

IRR99 makes it clear that all X-ray equipment that is being installed, moved or subject to a major maintenance procedure must be subject to a critical examination to show that it is electrically, mechanically and radiologically safe to use from a patient and staff perspective. Many dental practices are unaware that this is not only a legislative requirement for the installer, but also crucial for the practice in determining whether the set is functioning correctly before using it on patients. Previously published work has demonstrated that such faults can give rise to catastrophic failure and significant dose consequences. ${ }^{9}$

A number of dental practices had not arranged for the three-yearly set of routine checks on dental X-ray equipment to be carried out as specified in IRR99 and outlined in the Dental Guidance Notes to the Regulations. In other cases, previous records of such tests had been lost, typically when dental practices changed ownership. Without these routine checks being made, records being kept and subject to audit, either by the dental practice or the RPA/MPE, effective quality assurance and dose optimisation cannot be achieved.

The layout of dental surgeries with $\mathrm{X}$-ray equipment and the need (or lack of need) to provide additional shielding to the walls and doors in such surgeries has also proved a significant issue. Many dental practices did not have plans for new surgeries/equipment or modifications to existing equipment reviewed by an RPA before the work taking place.

Advice from an RPA at this stage can ensure that the control box and mains isolator switch for the X-ray set are positioned close to the usual operator's position, and that the position of the operator is not likely to be in the direction of the $\mathrm{X}$-ray beam or too close to the patient undergoing the exposure, which in some cases in this survey had been overlooked at the planning stage.

Experience has shown that many installers of dental X-ray equipment automatically assume the need for 'lead lining', often at considerable expense.

The report of the joint committee of the British Institute of Radiology (BIR) and the Institute of Physics and Engineering 
in Medicine (IPEM) entitled Radiation shielding for diagnostic $X$-rays ${ }^{10}$ makes it clear that unless the workload is very high or the dental surgery very small, additional shielding is unlikely to be needed. The report states that in most cases two sheets of standard plasterboard are likely to provide more than adequate protection to keep radiation doses in adjoining spaces well below the usual design dose constraint of $0.3 \mathrm{mSv}$ per year. These recommendations were made based on a dental set with a circular collimator of $60 \mathrm{~mm}$ diameter giving an entrance dose of 2 mGy. Given that recently-installed digital sets with rectangular collimators should produce exposures two to three times less than this, it is difficult to understand why some agencies are still recommending that: "the $\mathrm{X}$-ray beam should not be directed towards a light partition wall unless it can always be ensured that the adjacent area is not occupied' and 'should this not be practicable additional radiation shielding should be incorporated'. The BIR/IPEM report has recently been revised ${ }^{11}$ and shows that scattered radiation dose levels are in fact lower than had been previously considered. The key recommendation that plasterboard walls should provide sufficient protection for most intra-oral and panoramic workloads remains. This is an issue where direct assessment from an RPA for each individual dental practice would be able to clarify their shielding requirements.

These issues are even more important for dental practices that are considering the installation of CBCT equipment, where the significantly higher levels of radiation dose require improved room design and increased level of shielding during the planning and installation stage, followed up with increased training in equipment use and image interpretation. The frequency and complexity of QA checks are also significantly greater with CBCT equipment and demand increased involvement of the MPE to ensure patient radiation doses are optimised.
The data showed a wide range of equipment usage. The mean frequency of 33 exposures per week per set is somewhat higher than the figure of 20 exposures per week per set quoted in the BIR/ IPEM report on shielding. ${ }^{10,11}$ However, the fact that approximately $4 \%$ of the sets within the survey are being used for 100 or more exposures per week per set may well indicate that recommended criteria for patient selection such as that produced by the Faculty of General Dental Practitioners $\mathrm{UK}^{12}$ are not being followed and this needs further investigation.

One ionising radiation issue that has frequently been ignored is the topic of radon in the workplace. Although this is a naturally occurring source of ionising radiation exposure, it still needs to be addressed by the employer as part of their radiation risk assessment process. It is particularly an issue for dental practices in 'radon affected areas' and/or those with below-ground or poorly ventilated ground-floor work areas. Further information on this topic should be available from the Practice's RPA or from the HSE on its website (http://www.hse. gov.uk/radiation/ionising/radon.htm).

It should be noted that attendance at courses or online training in radiography and radiation protection is a requirement of the General Dental Council for continuing professional development (CPD) and that at least five hours is done in this subject every CPD cycle. Although such courses have been running for some time, it is clear that some dental practices remain largely unaware of staff and patient radiation protection issues and legislative compliance. It is to be hoped that as dental practitioners go through CPD cycles, their awareness improves.

\section{CONCLUSIONS}

Many dental practices still do not have either an RPA or an MPE to advise on patient protection issues. This goes against direct HSE policy that 'practices must consult and appoint a suitable radiation protection adviser about compliance with the IRR99'

Where no RPA/MPE appointment had been made, there was often a very low level of compliance with legislative requirements.

The active involvement of an MPE has the potential to reduce patient (and hence staff) radiation doses still further in many practices.

1. Health and Safety Executive. Compliance with IRR99: Action with professional organisations for dental practices and chiropractors. HSE Radiation Protection News 2010.

2. Ionising radiations regulations 1999. London: the Stationery Office, 1999. Online regulations available at http://www.legislation.gov.uk/uksi/1999/3232/ contents/made (accessed February 2013).

3. Ionising radiation (medical exposure) regula tions 2000. London: the Stationery Office, 2000 (amended 2006 and 2011)

4. National Radiological Protection Board. Guidance notes for dental practitioners on the safe use of $x$-ray equipment. London: NRPB, 2001. Online guidance available at http://www.hpa.org.uk/webc/ HPAwebFile/HPAweb_C/1194947310610 (accessed February 2013).

5. Hart D, Wall B F, Hillier M C, Shrimpton P C. HPACRCE-012: frequency and collective dose for medical and dental $x$-ray examinations in the UK. Didcot: Health Protection Agency Report, 2008. Online article available at http://www.hpa.org.uk/webc/ HPAwebFile/HPAweb_C/1287148001641 (accessed February 2013).

6. Hart D, Hillier M C, Wall B F. HPA-RPD-029: doses to patients from radiographic and flouroscopic $X$-ray imaging procedures in the UK - 2005 review. Didcot: Health Protection Agency Report, 2007.

7. Holroyd J R, Walker A. HPA-RPD-065: recommendations for the design of $x$-ray facilities and the quality assurance of dental cone beam CT (commuted tomography) systems. Didcot: Health Protection Agency Report, 2010. Online article available at http://www.hpa.org.uk/Publications/Radiation/ HPARPDSeriesReports/HPARPD065/ (accessed February 2013).

8. HPA Working Party on Dental Cone Beam CT Equipment. HPA-CRCE-010: guidance on the safe use of dental cone beam CT (commuted tomography) equipment. Didcot: Health Protection Agency Report, 2010. Online article available at http://www.hpa.org.uk/Publications/Radiation/ CRCEScientificAndTechnicalReportSeries/ HPACRCE010/ (accessed February 2013).

9. Malone, J F, Hone C. Analysis of a radiation incident with intraoral dental radiological equipment. Paper presented at a British Institute of Radiology meeting on radiation incidents in hospitals, 5 December, 1994.

10. British Institute of Radiology Institute of Physics and Engineering in Medicine. Radiation shielding for diagnostic $x$-rays. London: BIR/PEM Working Party, 2000.

11. Sutton D G, Martin C J, Williams J R, Peet D J. Radiation shielding for diagnostic $x$-rays. 2nd ed. London: British Institute of Radiology, 2012.

12. Faculty of General Dental Practitioners UK. Selection criteria for dental radiography. 2nd ed. London: Faculty of General Dental Practitioners UK (RCS), 2004. 